\title{
Higher pulmonary artery pressure in children than in adults upon fast ascent
}

\section{to high altitude}

\author{
S. Kriemler*\#, C. Jansen ", A. Linka", A. Kessel-Schaefer", M. Zehnder*, \\ T. Schürmann", M. Kohler ${ }^{+}$, K. Bloch $^{+}$and H.P. Brunner-La Rocca
}

ABSTRACT: The response of pulmonary artery pressure to high altitude has not been studied in children. It is also not known whether the individual response is hereditary. Therefore, the response of pulmonary artery pressure to high altitude was measured in pre-pubertal children in comparison to that in their biological fathers.

Echocardiography was performed at $450 \mathrm{~m}$ and over 3 days at $3,450 \mathrm{~m}$. Systolic pulmonary artery pressure was estimated from the pressure gradient of tricuspid regurgitation.

The increase in pulmonary artery pressure in children was greater than that in adults at day 1 of high altitude $(15.5 \pm 9.1$ versus $7.9 \pm 6.4 \mathrm{mmHg})$, but returned to adult levels on day 2 . The increase in pulmonary artery pressure from low to high altitude of each child correlated with that in the father.

Pre-pubertal children transiently develop greater pulmonary hypertension than their fathers when exposed to high altitude. The individual response of pulmonary pressure to high altitude seems to be at least partly hereditary.

KEYWORDS: Children, heredity, high altitude, high-altitude pulmonary oedema, pulmonary artery pressure

$t$ is increasingly popular for families to spend their holidays at high altitude, but the high altitude tolerance of children is barely known. The limited data available indicate that acute mountain sickness (AMS) is equally prevalent in adults and children [1]. However, the prevalence of high-altitude pulmonary oedema (HAPE) may differ between adults and children, despite some contradictory evidence [1]. Children residing at high altitude are more likely to develop HAPE [2, 3] when returning to high altitude after a lowland stay.

HAPE accounts for most deaths arising from high-altitude illness [4], and occurs in one per 10,000 population in Colorado (USA). Up to $10 \%$ of adults may be susceptible to HAPE [5]. The occurrence of HAPE depends upon individual susceptibility, rate of ascent and altitude reached. At an altitude of 4,500 m, HAPE occurs in 4-6\% after ascent within $<1$ day [6], and in up to $15 \%$ of adults at 5,500 m [7]. Although not everybody with increased hypoxia-induced pulmonary artery hypertension develops HAPE, the major pathophysiological mechanism in adults is exaggerated hypoxic pulmonary vasoconstriction with an abnormal increase in pulmonary artery pressure $(P$ pa) [8] and capillary pressure, which directly corresponds to HAPE susceptibility [9].

The underlying cause of HAPE in children is investigated very little. Children residing at high altitude developing HAPE after returning from low to high altitude may have an inherited predisposition to the development of pulmonary hypertension, as suggested for lowland adults [5], since their $P_{\text {pa }}$ rise is significantly greater than that of nonsusceptible controls when breathing a hypoxic gas mixture [3]. However, in addition to pulmonary artery hypertension, other mechanisms have been suggested, such as intercurrent viral infections leading to increased permeability of the blood-gas barrier $[10,11]$ or a history of transient perinatal hypertension, possibly as consequence of a persistent defect of nitric oxide synthesis [12].

Nevertheless, the response of $P$ pa to acute exposure to high altitude in children born at low altitude in comparison to that of adults is not known. Moreover, if a significant correlation of $P$ pa rise at high altitude existed between children and their parents, an inherited predisposition would probably be of importance. Therefore, 20

\section{AFFILIATIONS}

Institutes of *Exercise and Health Sciences, University of Basel, 'Cardiology, University Hospital Basel, Basel,

\#Exercise Physiology, Swiss Federal Institute of Technology Zurich, and +Pulmonary Division, University Hospital Zurich, Zurich, Switzerland.

CORRESPONDENCE

S. Kriemler

Institute of Exercise and Health Sciences

University of Basel

Brüglingen 33

4052 Basel

Switzerland

Fax: 41613778768

E-mail: susi.kriemler@unibas.ch

Received:

December 102007

Accepted after revision:

March 212008

SUPPORT STATEMENT

This study was supported by the Federal Council of Sports

(Magglingen, Switzerland). They had no role in the design and conduct of the study, or in the collection, management, analysis and interpretation of the data. There was no industry sponsorship.

STATEMENT OF INTEREST A statement of interest for this study can be found at www.erj.ersjournals.com/misc/ statements.shtml

European Respiratory Journal Print ISSN 0903-1936 Online ISSN 1399-3003 
lowland children and their fathers were studied at low altitude and over 3 days at high altitude in order to evaluate whether: 1) children differ from adults in their Ppa response to hypoxia; and 2) there is a hereditary influence on the magnitude of $P$ pa rise under hypoxic conditions.

\section{METHODS}

\section{Study participants}

In total, 20 healthy pre-pubertal children and their fathers were recruited through an announcement in the journal of the Swiss Alpine Club (Bern, Switzerland). Subjects with symptoms and signs of cardiovascular or pulmonary abnormalities, any sleeping problems revealed by history taking or a sleep study at low altitude, a previous history of perinatal pathology and a recent (within 2 months) history of respiratory infection were excluded from the study. None had a history of HAPE, but the majority had not been exposed to altitudes of $>4,000 \mathrm{~m}$. Subjects were not allowed to stay at altitudes of $>2,000 \mathrm{~m}$ during the 2 months prior to the study. On the preceding day and during all testing, subjects were not allowed to take any drugs other than acetaminophen. They abstained from any substances that might interfere with control of breathing, such as alcohol or caffeine, and had $\geqslant 8 \mathrm{~h}$ rest.night ${ }^{-1}$ prior to and during the study period. A physical examination of the cardiopulmonary system was performed at low altitude and daily at high altitude in order to ensure good general health. Height, weight and Tanner stage were assessed once at low altitude.

\section{Design}

Medical and physiological assessments were performed during a 4-h visit to the laboratory at low altitude (450 m; barometric pressure $718 \mathrm{mmHg}$ ) within 4 weeks prior to ascent, and at the Jungfraujoch research station at an altitude of $3,450 \mathrm{~m}$ (barometric pressure $509 \mathrm{mmHg}$ ), which they reached by train in $3 \mathrm{~h}$. Testing was performed $4-5 \mathrm{~h}$ after arrival on day 1 (HA1), during the afternoon of day 2 (HA2) after a mountain climb of $1-6 \mathrm{~h}$ to a maximal elevation of $4,010 \mathrm{~m}$, and in the morning of day 3 (HA3). The experimental protocol was approved by the ethics committee of the Swiss Federal Institute of Technology Zurich (Zurich, Switzerland) and all of the participants provided written informed consent.

\section{Measurements}

Cardiac frequency was measured using a cardiac frequency monitor (Polar Vantage XL; Polar, Kempele, Finland) and arterial oxygen saturation by pulse oximetry (OxiMax N-595; Nellcor, Leuag, Stans, Switzerland) of the finger after a 15-min rest sitting in a comfortable chair. The mean of each over a 5min period was taken. Systemic blood pressure (BP) was measured prior to echocardiography after $\sim 15$ min of lying quietly; the mean of three consecutive measurements was calculated. Doppler echocardiography was performed by means of an integrated colour Doppler system using a 4.0$\mathrm{MHz}$ transducer (Toshiba Aplio 80; Toshiba, Oetwil am See, Switzerland). For assessment of the peak pressure gradient between the right ventricle and right atrium, tricuspid valve regurgitation was first located by colour Doppler imaging in the four-chamber view or modified four-chamber view, if required. The peak flow velocity of the transtricuspid regurgitant jet ( $\left.V^{\prime} T R\right)$ then was measured using continuous-wave Doppler. Based on the peak velocity, the transtricuspid pressure gradient (DPTR) was calculated using the simplified Bernoulli equation $\left(\mathrm{DPTR}=4 V^{\prime} \mathrm{TR}^{2}\right)$. Diastolic $P$ pa was estimated from the pressure gradient across the pulmonary valve at end of diastole, after locating the pulmonary insufficiency jet in the parasternal view using colour Doppler recordings. Additionally, pulmonary vascular resistance (PVR) was estimated using the following formula [13]:

$$
\mathrm{PVR}=\frac{10 V^{\prime} \mathrm{TR}}{\mathrm{VTIRVOT}}+0.16
$$

where VTIRVOT is the velocity-time integral of systolic flow in the right ventricular outflow tract using pulse-wave Doppler recording. Finally, the acceleration time relative to ejection time was calculated as an indirect measure of mean Ppa [14].

All recordings were stored on magneto-optical disks and independently analysed off-line by three investigators using the mean of three consecutive heart beats. In the case of disagreement (i.e. $>10 \%$ ), recordings were reanalysed by the three investigators together. Consensus was found for all measurements.

\section{Acute mountain sickness}

AMS was assessed by means of a scoring system for its symptoms and signs using the Environmental Symptoms Questionnaire (cerebral symptoms of AMS (AMS-C) score) [15] and also the Lake Louise score [16]. An AMS-C score of $\geqslant 0.70$ and a Lake Louise score of $\geqslant 5$ were defined as diagnostic for AMS.

\section{Statistics}

Data are presented as mean $\pm S D$ unless otherwise stated. Changes from low to high altitude and between groups were analysed using a two-way (low to high altitude; children versus adults) general linear model for repeated measures with post hoc testing, including adjustment according to Bonferroni. The differences in the response from low to high altitude between children and adults were examined using the interaction term (response to altitude groups) of the above-mentioned model. Comparison of individual values between groups was performed using the t-test for paired samples (unadjusted for baseline measurement; adjusted for multiple comparisons at high altitude). In order to test a significant relationship of response to high altitude between children and their fathers, pairs of father and child were used as covariate in the general linear model. Correlations between variables were examined using Pearson's or Spearman's r, as indicated.

\section{RESULTS}

The baseline characteristics of the subjects are shown in table 1. As expected, the 9-12-yr-old children exhibited a lower BP and higher cardiac frequency at low altitude than their fathers. $P$ pa did not differ significantly between the two groups and the calculated PVR was identical. However, $P$ pa relative to systemic pressure was higher in children at low altitude. Arterial oxygen saturation was slightly, although significantly, higher in children.

One female child developed severe AMS and an exaggerated $P$ pa after $4 \mathrm{~h}$ of altitude exposure and had to be excluded from further analysis because of treatment with dexamethasone. A 


\begin{tabular}{|c|c|c|}
\hline & Children & Adults \\
\hline Age yrs & $10.7 \pm 1.1$ & $44.0 \pm 4.0$ \\
\hline Height $\mathrm{cm}$ & $143 \pm 8$ & $179 \pm 7$ \\
\hline Weight kg & $33 \pm 6$ & $74 \pm 7$ \\
\hline Systolic BP mmHg & $94 \pm 7$ & $125 \pm 13^{*}$ \\
\hline Diastolic BP mmHg & $46 \pm 7$ & $65 \pm 9^{*}$ \\
\hline Cardiac frequency beats $\cdot \min ^{-1}$ & $73 \pm 9$ & $61 \pm 8^{*}$ \\
\hline Systolic Ppa mmHg & $21.9 \pm 3.2$ & $24.1 \pm 4.5$ \\
\hline Diastolic Ppa mmHg & $6.9 \pm 1.4$ & $7.6 \pm 2.3$ \\
\hline PVR dyn $\cdot \mathrm{cm}^{-5} \cdot \mathrm{s}$ & $114 \pm 19$ & $113 \pm 15$ \\
\hline $\mathrm{Sa}, \mathrm{O}_{2} \%$ & $98.0 \pm 1.0$ & $97.3 \pm 1.0^{*}$ \\
\hline
\end{tabular}

Data are presented as mean \pm SD. BP: blood pressure; $P$ pa: pulmonary artery pressure; PVR: pulmonary vascular resistance; $\mathrm{Sa}, \mathrm{O}_{2}$ : arterial oxygen saturation. *: $p<0.05$ versus children.

male child completed the measurements on day 1 and then went home with their father because of home sickness. In one child, the Doppler signal across the tricuspid valve was insufficient for analysis on day 3.

The increase in Ppa was significantly greater in children than in adults on day 1 (absolute increase $15.3 \pm 8.7$ versus $7.7 \pm$ $6.4 \mathrm{mmHg}$, respectively, $\mathrm{p}=0.004$, fig. 1; relative increase $91 \pm 54$ versus $45 \pm 42 \%, \mathrm{p}=0.003$ ), although the altitudeinduced fall in arterial oxygen saturation did not differ between the groups (table 2). Systolic BP increased in children but not in adults $(p=0.03$; table 2$)$, but this increase was smaller than that in systolic $P$ pa $(\mathrm{p}<0.01)$. On average, systolic $P$ pa relative to systolic BP on day 1 at high altitude increased more in children than in adults $(0.15 \pm 0.09$ versus $0.07 \pm 0.05$, $\mathrm{p}=0.002)$. In addition, diastolic Ppa $(5.4 \pm 3.2$ versus $1.6 \pm$ $2.8 \mathrm{mmHg}, \mathrm{p}=0.04)$ and calculated PVR increased $(40 \pm 27$ versus $\left.25 \pm 21 \mathrm{dyn} \cdot \mathrm{s} \cdot \mathrm{cm}^{-5}, \mathrm{p}=0.02\right)$, and acceleration time relative to the ejection time of Doppler flow in the right ventricular outflow tract decreased significantly more in children at high altitude $(0.13 \pm 0.08$ versus $0.08 \pm 0.05$, $\mathrm{p}=0.04$ ), consistent with the findings of systolic Ppa (table 3). Interestingly, the increase in systolic $P$ pa from low to high altitude on day 1 of each child correlated significantly with the percentage increase of their father $(r=0.57, p=0.009$, fig. 2).

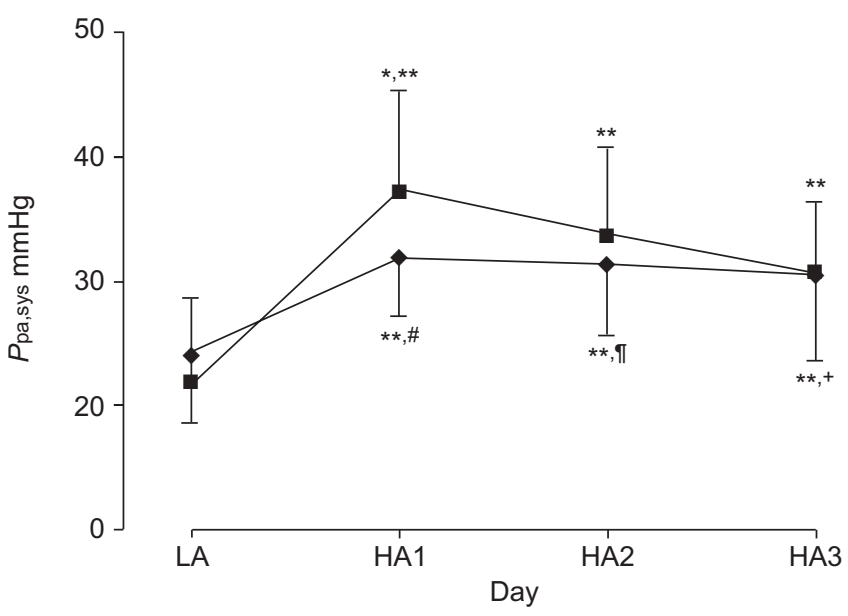

FIGURE 1. Effect of high altitude ( $\mathrm{HA} ; 3,450 \mathrm{~m})$ exposure on systolic pulmonary artery pressure (Ppa,sys) in children $(\boldsymbol{\square})$ and adults $(\boldsymbol{)})$ on days 1,2 and 3 of HA. Data are presented as mean \pm SD. In both groups, hypoxia induced a significant increase in $P$ pa,sys compared with normoxia. LA: low altitude (450 m). *: $p<0.05$ versus adults; **: $p<0.01$ versus normoxia; ${ }^{*}: p=0.009 ; \quad$ : $p=0.03$; +: $p=0.30$ versus children's response from baseline (change in $P$ pa,sys).

This relationship was also significant if tested using a multivariate general linear model for repeated measures $(p=0.005)$. A similar relationship was seen regarding the calculated PVR $(p=0.02)$, and nonsignificant trends were seen regarding diastolic $P_{\mathrm{pa}}(\mathrm{p}=0.22)$ and acceleration time relative to ejection time $(p=0.12)$. Cardiac frequency increased significantly more in children than in adults $(p=0.002$, table 2$)$. This increase in cardiac frequency correlated significantly with the increase in systolic $P$ pa in both groups $(\mathrm{r}=0.46, \mathrm{p}=0.003)$, as did the increase in systolic BP $(r=0.35, p=0.03)$. The decrease in arterial oxygen saturation also, but to a lesser extent, correlated with the increase in $P$ pa in the whole group $(\mathrm{r}=-0.31, \mathrm{p}=0.05)$.

On days 2 and 3, systolic Ppa gradually decreased in children, whereas it remained stable in adults (fig. 1). Nevertheless, the overall response over the 3 days including only those who completed the whole study ( $n=17$ pairs) showed a significantly different response between children and adults in the general linear model for repeated measures $(p=0.02)$, with significant correlation between related pairs $(p=0.02)$. The relationship of systolic $P$ pa and systolic BP was persistently greater in children than in adults $(p<0.05$, table 3$)$, with a

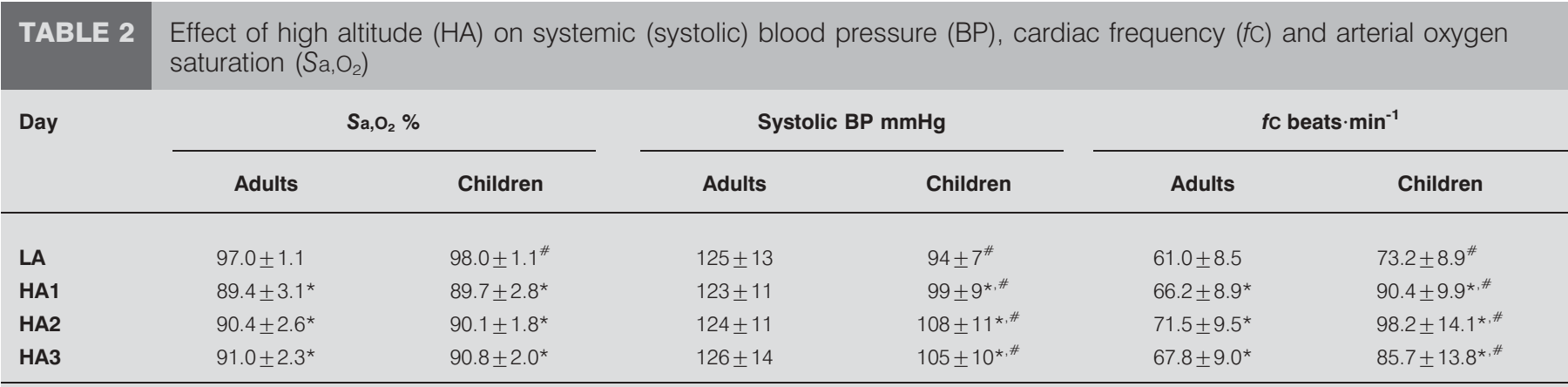

Data are presented as mean $\pm \mathrm{SD}$. LA: low altitude. *: $p<0.05$ versus baseline; ${ }^{*}: \mathrm{p}<0.05$ versus adults. 


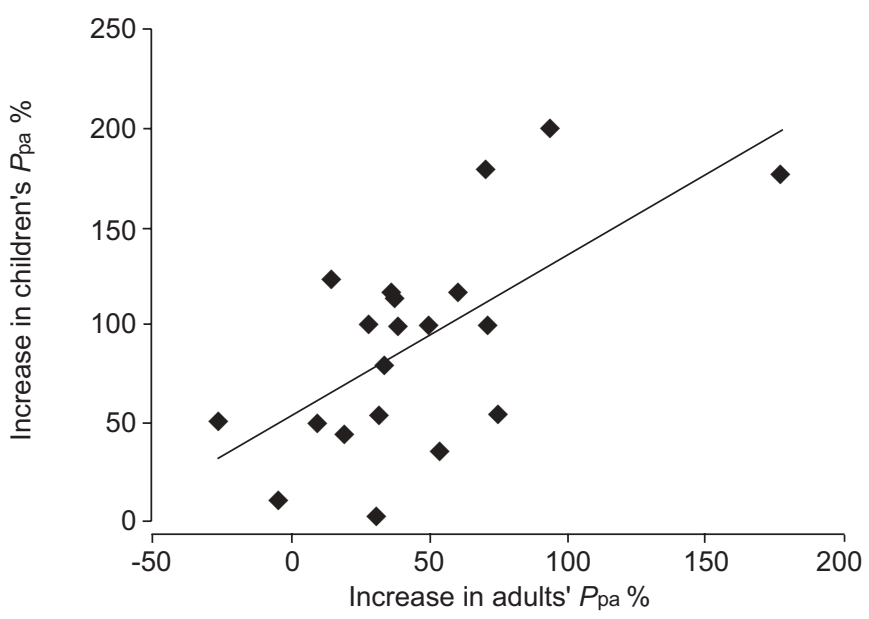

FIGURE 2. Relationship between the increase in systolic pulmonary artery pressure $(\mathrm{Ppa})$ from low altitude to day 1 of high altitude of each child with that of the father. — : line of best fit. Spearman's $r=0.57 ; p<0.009$.

significant relationship in related pairs $(p=0.03)$. In addition, the estimated PVR remained significantly more elevated in children throughout the study, as did diastolic Ppa on day 2 (table 3).

At high altitude, AMS by AMS- $C$ score occurred in 10 children and six adults $(p=0.20)$, and in eight children and seven adults $(p=0.74)$ when using the Lake Louise score. Overall, the increase in systolic Ppa was greater in the subjects who developed AMS using the AMS-C score and combining children and adults $(15 \pm 9$ versus $9 \pm 7 \mathrm{mmHg}, p=0.04)$, but this difference was nonsignificant when using the Lake Louise score (14 \pm 8 versus $10 \pm 8 \mathrm{mmHg}, \mathrm{p}=0.19)$. Arterial oxygen saturation in those with and without AMS did not differ significantly for the whole group $(90.0 \pm 2.8$ versus $88.9 \pm 3.1 \%, \mathrm{p}=0.26)$ nor when children and adults were analysed separately.

\section{DISCUSSION}

The increase in Ppa was greater in children than in adults on the first day of high altitude exposure. This cannot be attributed to lower arterial oxygen saturations since the degree of altitude-induced hypoxaemia did not differ between the two groups. Systemic BP and cardiac frequency also increased more in children than in adults, suggesting a greater response of the sympathetic nervous system in children with exposure to hypoxia. The similar $P$ pa at low altitude in both groups and the fact that healthy children without perinatal complications were selected suggest that the exaggerated pulmonary vasoconstrictor response was related to an adaptive rather than a structural difference. This adaptive difference was not selective of the pulmonary circulation and also included the systemic circulation, but the response of the pulmonary arterial system clearly exceeded that of the systemic vasculature.

The autonomic nervous system plays an important role in mediating many of the physiological adjustments to hypoxia, leading to increased cardiac frequency and cardiac output to counterbalance the decreased oxygen availability from air [17]. Therefore, the sympathetic nervous system may also contribute to an exaggerated pulmonary pressure rise, and is one of the suggested pathophysiological mechanisms of hypoxiainduced pulmonary hypertension and HAPE. Increased sympathetic nerve activity in skeletal muscles, which is directly related to $P$ pa and occurs prior to the development of HAPE, has indeed been shown in HAPE-susceptible subjects [18]. Since the present study was performed at a moderate altitude of $3,450 \mathrm{~m}$, at which HAPE rarely occurs in adults [19], none of the present subjects developed HAPE. Nevertheless, a rather large increase in Ppa accompanied by severe AMS, both linked to the development of HAPE in adults [4], was found in one female child within $\sim 3 \mathrm{~h}$ following exposure to high altitude [20]. The severe AMS required immediate treatment including dexamethasone, after which the child was noted to show a reduced $P$ pa. Although reduced $P$ pa has been shown to effectively prevent the development of HAPE in adults [21], it is not possible to know whether HAPE would have developed in this child without treatment.

Importantly, the $P$ pa found in the children on the first day corresponded to that measured in HAPE-insusceptible adults at an altitude of $4,559 \mathrm{~m}$, i.e. $>1,000 \mathrm{~m}$ higher than in the present study [21]. However, there is no evidence to date that children are more susceptible to the development of HAPE than adults. The children showed a gradual decrease in $P$ pa on days 2 and 3, converging with the $P$ pa in the adults, although some of the indirect measures of pulmonary vasoconstriction remained elevated. Nevertheless, adaptation to altitude seems

\section{TABLE 3 Effect of high altitude (HA) on Doppler echocardiographic pulmonary haemodynamic parameters}

\begin{tabular}{|c|c|c|c|c|c|c|c|c|}
\hline Day & \multicolumn{2}{|c|}{ Calculated PVR dyn $\cdot \mathbf{s} \cdot \mathrm{cm}^{-5}$} & \multicolumn{2}{|c|}{$\mathrm{DPPI} \mathrm{mmHg}$} & \multicolumn{2}{|c|}{ AcT/ET } & \multicolumn{2}{|c|}{ Systolic $P$ pa/BP } \\
\hline HA1 & $138 \pm 18^{*}$ & $155 \pm 22^{*, \#}$ & $6.2 \pm 4.2$ & $7.6 \pm 3.2^{*}$ & $0.37 \pm 0.06^{*}$ & $0.33 \pm 0.07^{\star}$ & $0.26 \pm 0.04^{*}$ & $0.38 \pm 0.09^{*, \#}$ \\
\hline HA2 & $138 \pm 25^{*}$ & $163 \pm 32^{*, \#}$ & $4.7 \pm 3.1$ & $7.8 \pm 4.7^{*}$ & $0.33 \pm 0.05^{\star}$ & $0.32 \pm 0.08^{*}$ & $0.25 \pm 0.05^{*}$ & $0.31 \pm 0.07^{*, \#}$ \\
\hline HАЗ & $141 \pm 33^{*}$ & $154 \pm 38^{*}$ & $4.4 \pm 3.1$ & $5.7 \pm 2.6$ & $0.34 \pm 0.06^{*}$ & $0.33 \pm 0.06^{*}$ & $0.24 \pm 0.06^{*}$ & $0.29 \pm 0.04^{*, \#}$ \\
\hline
\end{tabular}

Data are presented as mean \pm SD. PVR: pulmonary vascular resistance; DPPI: pressure gradient across pulmonary valve at end diastole; AcT: acceleration time in right ventricular outflow tract; ET: ejection time in right ventricular outflow tract; Ppa: pulmonary artery pressure; BP blood pressure; LA: low altitude. *: $p<0.05$ versus baseline;

$\#: \mathrm{p}<0.05$ versus adults. 
to occur within 2 or 3 days in children, emphasising the importance of slow ascent. This seems particularly important since rapid exposure to high altitude, as is often the case during holidays, is one of the most important risk factors for the development of HAPE [7].

Another important aspect of the present study is that evidence was found of a hereditary component of the individual response of $P$ pa to high-altitude induced hypoxia. There was significant correlation of the response of the $P$ pa to high altitude of each child with that of their father, which is a novel finding. However, some forms of pulmonary arterial hypertension are known to be hereditary [22], and, in families with known pulmonary arterial hypertension, gene carriers exhibit a substantially greater risk of exercise-induced pulmonary hypertension [23]. Given the fact that only one parent of each child (i.e. half of the genetic information) was investigated, the results of the present study suggest significant hereditary determination of the individual response of the pulmonary vasculature to hypoxia, but the magnitude remains to be investigated.

Finally, the present study suggests that stimulation of sympathetic tone may contribute to the excessive pulmonary hypertensive response to hypoxia. This may be particularly important for the excessive rise in $P$ pa in children since they showed larger increases in both cardiac frequency and systolic BP. Therefore, the increased $P$ pa in children in the present study is probably caused by a combination of increased flow, direct sympathetic vasoconstriction, as shown in the animal model [24], and, to a minor extent, direct hypoxia-induced vasoconstriction. A disproportionate increase in sympathetic tone has also been described in individuals developing HAPE [18], but previous results have not been uniform [5]. Other factors may additionally contribute to the individual response, such as a reduced nitric oxide (NO) availability or increased endothelin 1, angiotensin II or arachidonic acid metabolites [5]. $\mathrm{NO}$, in particular, has been repeatedly suggested as an important factor [5]. Interestingly, there is some evidence that children might exhibit reduced NO production in the pulmonary circulation compared with adults [25, 26], but the present study did not investigate the potential involvement of NO and other factors. In addition, the evidence of increased sympathetic tone as a potentially contributory factor is only indirect. Thus further studies are required in order to address this issue in more detail.

There are some limitations that may apply to the present study. Mothers were not included as the influence of the menstrual cycle on the pulmonary vascular response to high altitude is not well defined. In addition, it would have been difficult to recruit the majority of the mothers since only children within a very narrow age range were included. Furthermore, all of the haemodynamic measurements were obtained noninvasively, giving rise to some level of uncertainty. Finally, sympathetic tone was not directly measured. Additional analyses, such as urine catecholamine levels or catecholamine kinetics, would have supported the increased sympathetic tone.

In conclusion, pre-pubertal children exhibit a greater increase in pulmonary artery pressure upon high-altitude induced hypoxia than do their fathers, an effect which was transient and limited to the first day of altitude exposure. The more pronounced pulmonary vascular response of children is, in part, related to greater sympathetic tone and modulated by a hereditary component. There is no evidence to date that the excessive increase in pulmonary artery pressure in children may predispose them to high-altitude pulmonary oedema.

\section{ACKNOWLEDGEMENTS}

The authors would like to thank all of the participants, and especially the children, for their valuable contribution to the present study. The authors thank Toshiba (Oetwil am See, Switzerland) for making available the ultrasound equipment and Leuag (Stans, Switzerland) for making available the pulse oximeter. The authors would also like to thank all of the team members of the High Altitude Research Station Jungfraujoch (Switzerland) for their competent and extraordinary support.

\section{REFERENCES}

1 Pollard AJ, Niermeyer S, Barry P, et al. Children at high altitude: an international consensus statement by an ad hoc committee of the International Society for Mountain Medicine, March 12, 2001. High Alt Med Biol 2001; 2: 389-403.

2 Scoggin CH, Hyers TM, Reeves JT, Grover RF. High-altitude pulmonary edema in the children and young adults of Leadville, Colorado. N Engl J Med 1977; 297: 1269-1272.

3 Fasules JW, Wiggings JW, Wolfe R. Increased lung vasoreactivity in children from Leadville, Colorado, after recovery from high-altitude pulmonary edema. Circulation 1985; 72: 957-962.

4 Hackett PH, Roach RC. High-altitude illness. N Engl J Med 2001; 345: 107-114.

5 Bartsch P, Mairbaurl H, Maggiorini M, Swenson ER. Physiological aspects of high-altitude pulmonary edema. J Appl Physiol 2005; 98: 1101-1110.

6 Maggiorini M, Buhler B, Walter M, Oelz O. Prevalence of acute mountain sickness in the Swiss Alps. BMJ 1990; 301: 853-855.

7 Singh I, Kapila CC, Khanna PK, Nanda RB, Rao BD. Highaltitude pulmonary oedema. Lancet 1965; 1: 229-234.

8 Scherrer U, Vollenweider L, Delabays A, et al. Inhaled nitric oxide for high-altitude pulmonary edema. $N$ Engl J Med 1996; 334: 624-629.

9 Maggiorini M, Melot C, Pierre S, et al. High-altitude pulmonary edema is initially caused by an increase in capillary pressure. Circulation 2001; 103: 2078-2083.

10 Durmowicz AG, Noordeweir E, Nicholas R, Reeves JT. Inflammatory processes may predispose children to highaltitude pulmonary edema. J Pediatr 1997; 130: 838-840.

11 Das BB, Wolfe RR, Chan KC, Larsen GL, Reeves JT, Ivy D. High-altitude pulmonary edema in children with underlying cardiopulmonary disorders and pulmonary hypertension living at altitude. Arch Pediatr Adolesc Med 2004; 158: 1170-1176.

12 Sartori C, Allemann Y, Trueb L, Delabays A, Nicod P, Scherrer U. Augmented vasoreactivity in adult life associated with perinatal vascular insult. Lancet 1999; 353: 2205-2207.

13 Abbas AE, Fortuin FD, Schiller NB, Appleton CP, Moreno CA, Lester SJ. A simple method for noninvasive estimation of pulmonary vascular resistance. J Am Coll Cardiol 2003; 41: 1021-1027. 
14 Huez S, Retailleau K, Unger $P$, et al. Right and left ventricular adaptation to hypoxia: a tissue Doppler imaging study. Am J Physiol Heart Circ Physiol 2005; 289: H1391-H1398.

15 Sampson JB, Cymerman A, Burse RL, Maher JT, Rock PB. Procedures for the measurement of acute mountain sickness. Aviat Space Environ Med 1983; 54: 1063-1073.

16 Roach RC, Bartsch P, Hackett PH, Oelz O. The Lake Louise acute mountain sickness scoring system. In: Sutton JR, Houston CS, Coates G, eds. Hypoxia and Molecular Medicine. Burlington, Queens City Press, 1993; pp. 272-274.

17 Seals DR, Johnson DG, Fregosi RF. Hypoxia potentiates exercise-induced sympathetic neural activation in humans. J Appl Physiol 1991; 71: 1032-1040.

18 Duplain H, Vollenweider L, Delabays A, Nicod P, Bartsch P, Scherrer U. Augmented sympathetic activation during short-term hypoxia and high-altitude exposure in subjects susceptible to high-altitude pulmonary edema. Circulation 1999; 99: 1713-1718.

19 Sophocles AM, Bachman J. High-altitude pulmonary edema among visitors to summit county, Colorado. J Fam Pract 1983; 17: 1015-1017.

20 Kriemler S, Kohler M, Zehnder M, Bloch KE, Brunner-La Rocca H. Successful treatment of severe acute mountain sickness and excessive pulmonary hypertension with dexamethasone in a prepubertal girl. High Alt Med Biol 2006; 7: 256-261.

21 Maggiorini M, Brunner-La Rocca H-P, Peth S, et al. Both tadalafil and dexamethasone may reduce the incidence of high-altitude pulmonary edema: a randomized trial. Ann Intern Med 2006; 145: 497-506.

22 McLaughlin VV, McGoon MD. Pulmonary arterial hypertension. Circulation 2006; 114: 1417-1431.

23 Grunig E, Janssen B, Mereles D, et al. Abnormal pulmonary artery pressure response in asymptomatic carriers of primary pulmonary hypertension gene. Circulation 2000; 102: 1145-1150.

24 Shirai M, Matsukawa K, Nishiura N, Kawaguchi AT, Ninomiya I. Changes in efferent pulmonary sympathetic nerve activity during systemic hypoxia in anesthetized cats. Am J Physiol 1995; 269: R1404-R1409.

25 Buchvald F, Baraldi E, Carraro S, et al. Measurements of exhaled nitric oxide in healthy subjects age 4 to 17 years. $J$ Allergy Clin Immunol 2005; 115: 1130-1136.

26 Avital A, Uwyyed K, Berkman N, Bar-Yishay E, Godfrey S, Springer C. Exhaled nitric oxide is age-dependent in asthma. Pediatr Pulmonol 2003; 36: 433-438. 